\title{
O VOLUME DE EXERCÍCIOS RESISTIDOS INFLUENCIA A REATIVIDADE DA PRESSÃO ARTERIAL AO ESTRESSE
}

\author{
THE VOLUME OF RESISTANCE EXERCISES INFLUENCES BLOOD PRESSURE REACTIVITY TO STRESS
}

\author{
EL VOLUMEN DE EJERCICIOS DE FUERZA INFLUYE EN LA REACTIVIDAD DE LA \\ PRESIÓN ARTERIAL AL ESTRÉS
}

Mauro Fernando Lima da Silva ${ }^{1,5}$ (Educador Fisico)

Carmen Silvia Grubert Campbell ${ }^{5}$ (Educadora Física)

Aline de Freitas Brito ${ }^{6}$ (Educadora Física)

Alexandre Sérgio Silva² (Educador Físico)

Marcos Antônio Pereira dos Santos ${ }^{3}$ (Educador Físico)

Marcelle Napoleão do Rêgo Formiga ${ }^{4}$ (Psicóloga)

Sergio Rodrigues Moreira (Educador Físico)

Herbert Gustavo Simões ${ }^{5}$

(Educador Físico)

1. Universidade Federal o Piauí; Departamento de Educação Física; Teresina, PI, Brasil.

2. Universidade Federal da Paraíba; Departamento de Educação Física; Laboratório de Estudos do Treinamento Físico Aplicado ao Desempenho e à Saúde; João Pessoa, PB, Brasil.

3. Universidade Federal do Piauí; Departamento de Biofísica e

Fisiologia; Teresina, PI, Brasil.

4. Faculdades de Ciências Médicas do Estado do Piauí; Departamento de Psicologia; Teresina, PI, Brasil.

5. Universidade Católica de Brasília, Brasília-DF, Brasil.

6. Universidade Federal do Piauí;

Departamento de Métodos e

Técnicas de Ensino; Teresina,

PI, Brasil.

7. Universidade Federal do Vale do São Francisco - UNIVASF; Petrolina, PE, Brasil.

\section{Correspondência:}

Rua São Pedro, 965, Centro. Teresina,

Piauí, Brasil. 64001-260

mauropetman@gmail.com

\section{RESUMO}

Introdução: A hiper-reatividade da pressão arterial (PA) ao estresse pode estar associada ao desenvolvimento da hipertensão arterial. Porém a realização prévia de exercícios físicos tem se mostrado eficaz em atenuar esta elevação da PA ao estresse subsequente. Objetivo: Verificar o efeito do volume da sessão de exercícios resistidos (ER) sobre a reatividade da PA (RV) ao estresse induzido. Métodos: Quarenta adultos jovens normotensos foram submetidos a três sessões experimentais em ordem randomizada: Sessão com 2 séries de ER (S2); Sessão com 4 séries de ER (S4); e Sessão controle sem exercícios (SC). S2 e S4 foram realizadas à 70\% de uma repetição máxima e compostas por seis exercícios com 10 repetições cada (intervalo de 90s entre séries). Aos 10 minutos de recuperação das sessões experimentais, os voluntários foram submetidos ao Cold Pressor Test (CPT), que é um protocolo de estresse no qual a mão direita é submergida em água gelada $\left(4\right.$ a $5^{\circ} \mathrm{C}$ ) durante 1 minuto para determinar a RV. A PA sistólica (PAS), diastólica (PAD), média (PAM) e frequência cardíaca (FC) foram mensuradas no repouso pré-sessões experimentais (20 minutos) e imediatamente após o CPT. Resultados: Em resposta ao estresse induzido pelo CPT a FC não diferenciou após sessões experimentais (SC: $98 \pm 9$ vs. S2: $107 \pm 12$ vs. S4: $113 \pm 13$ bpm; $P>0,05)$. Entretanto, após a sessão de ER de maior volume, ocorreu atenuação da RV na PAS (SC: $145 \pm 18$ vs. $S 2: 142 \pm 16$ vs. $54: 133 \pm 15$ mmHg; $P<0,05), P A D$ (SC: $96 \pm 13$ vs. $S 2: 90 \pm 14$ vs. $54: 86 \pm 12$ mmHg; $P<0,05$ ) e PAM (SC: $89 \pm 10$ vs. S2: $91 \pm 5$ vs. S4: $91 \pm 4$ mmHg; $P<0,05$ ). Conclusão: $O$ maior volume (4 séries vs. 2 séries) na sessão de ER resultou em atenuação da reatividade da PA frente ao estresse induzido.

Palavras-chave: treinamento de resistência, pressão arterial, estresse fisiológico.

\section{ABSTRACT}

Introduction: The hyper-reactivity of blood pressure (BP) to stress can be associated with the development of arterial hypertension. However, performing physical exercises beforehand has proven to be effective in attenuating this $B P$ elevation in response to subsequent stress. Objective: Verify the effect of the volume of resistance exercise session (RE) on blood pressure reactivity (VR) to induced stress. Methods: Forty normotensive young adults underwent three experimental sessions randomly: Session of 2 series of RE (S2); Session of 4 series of RE (S4); and a Control session without exercises (CS). S2 and S4 were performed at 70\% of one repetition maximum and composed of six exercises with 10 repetitions each (90s interval between series). At the $10^{\text {th }}$ minute of recovery from experimental sessions, the volunteers underwent a Cold Pressor Test (CPT), which is a stress protocol where the right hand is immersed in cold water (4-5 $\mathrm{C}$ ) for 1 minute to determine VR. Systolic BP (SBP), diastolic (DBP), mean (MBP) and heart rate (HR) were measured at rest before experimental sessions (20 minutes) and immediately after CPT. Results: In response to the stress induced by CPT the HR did not differ between experimental sessions (CS: $98 \pm 9$ vs. S2: $107 \pm 12$ vs. S4: $113 \pm 13$ bpm, P>0.05). However, after a session of higher volume RE, attenuation of VR was observed for SBP (CS: $145 \pm 18$ vs. S2: $142 \pm 16$ vs. S4: $133 \pm$ $15 \mathrm{mmHg} ; P<0.05)$ ), DBP (CS: $96 \pm 13$ vs. S2: $90 \pm 14$ vs. S4: $86 \pm 12 \mathrm{mmHg}$; $P$ <0.05) and MBP (CS: $89 \pm 10$ vs. S2: $91 \pm$ 5 vs. $54: 91 \pm 4 \mathrm{mmHg}, P<0.05$ ). Conclusion: The higher volume (4 series vs. 2 series) of resistance exercises resulted in attenuation of blood pressure reactivity to stress induction.

Keywords: resistance training, arterial pressure, stress physiological.

\section{RESUMEN}

Introducción: La hiperreactividad de la presión arterial (PA) al estrés puede estar asociada al desarrollo de la hipertensión arterial. Sin embargo, la realización previa de ejercicios físicos se mostró eficaz para atenuar la elevación de la PA frente al estrés subsecuente. Objetivo: Verificar el efecto del volumen de la sesión de ejercicios de fuerza (EF) en la reactividad de la presión arterial (RV) frente al estrés inducido. Métodos: Cuarenta jóvenes adultos normotensos se sometieron a tres sesiones experimentales, al azar: Sesión con 2 series de EF (S2); Sesión con 4 series EF (S4) y; Sesión de control sin ejercicios (SC). S2 y S4 se realizaron a 70\% de una repetición máxima y compuesta de seis ejercicios con 10 repeticiones cada uno (intervalo de 90s entre las series). A los 10 minutos de la recuperación de las sesiones experimentales, los voluntarios fueron sometidos a prueba de frío presor (Cold Pressor Test - CPT), un protocolo de estrés en el cual la mano derecha es inmersa en agua con temperatura de $4 a 5^{\circ} \mathrm{C}$ durante 1 minuto para determinar la RV. La PA sistólica (PAS), diastólica (PAD), media (PAM) y frecuencia cardiaca (FC) se midieron en reposo antes de las sesiones experimentales (20 minutos) e inmediatamente después de la CPT. Resultados: En respuesta al estrés 
inducido por CPT, FC no se diferenció después de las sesiones experimentales (SC: $98 \pm 9$ vs. S2: 107 \pm 12 vs. S4: $113 \pm 13$ bpm; $P>0,05)$. Sin embargo, después de la sesión de EF de mayor volumen, hubo atenuación de la RV en la PAS (SC: $145 \pm 18$ vs. S2: $142 \pm 16$ vs. S4: $133 \pm 15 \mathrm{mmHg} ; p<0,05$ ), PAD (SC: $96 \pm 13$ vs. $\$ 2: 90 \pm 14$ vs. $S 4: 86 \pm 12 \mathrm{mmHg} ; p<0,05$ ) y PAM (SC: $89 \pm 10$ vs. S2: $91 \pm 5$ vs. S4: $91 \pm 4 \mathrm{mmHg} ; p<0,05$ ). Conclusión: El mayor volumen (4 series vs. 2 series) de EF en la sesión resultó en la atenuación de la reactividad para PA frente al estrés inducido.

Palabras clave: entrenamiento de resistencia, presión arterial, estrés fisiológico.

\section{INTRODUÇÃO}

A manutenção crônica de altos níveis de estresse pode estar associada ao desenvolvimento de doenças ${ }^{1}$, como insuficiência cardíaca², aterosclerose $^{3}$, isquemia ${ }^{4}$ e hipertensão arterial $(\mathrm{HA})^{5}$. A HA tem sido associada com uma aumentada reatividade vascular durante e após períodos agudos de estresse induzido ${ }^{6,7}$. Estudos anteriores ${ }^{8,9}$ evidenciaram aumentos no débito cardíaco, na vasoconstricção, na resistência vascular periférica, na atividade nervosa simpática e, por conseguinte, elevações anormais na pressão arterial em humanos que se encontravam em condições de estresse.

Embora a manutenção da pressão arterial em valores considerados normais muitas vezes requer modificações no estilo de vida, principalmente hábitos nutricionais saudáveis e a prática regular de exercícios físicos $^{10-11}$, sabe-se que uma única sessão de exercício físico pode ser uma alternativa eficaz para atenuar a resposta pressórica ao estresse cardiovascular subsequente ${ }^{4,11,12}$

O exercício físico pode promover aumento os níveis circulantes de endorfinas ${ }^{5-12}$, aumento da atividade autonômica parassimpática em repouso e períodos de recuperação pós-exercícios ${ }^{12}$, bem como, diminuição das concentrações séricas e locais de noradrenalina ${ }^{2}$ e aumento da biodisponibilidade de óxido nítrico. Ullman et al. ${ }^{13}$, demostraram que indivíduos normotensos com hiper-reatividade da pressão arterial ao estresse, quando submetidos a um programa de treinamento aeróbio, reduzem seus níveis pressóricos em resposta a um teste de estresse cardiovascular.

Apesar dos importantes benefícios apontados pelos estudos previamente citados, a maioria foi realizada utilizando-se de exercícios aeróbios. Pelo que temos conhecimento, apenas um estudo realizado especificamente com exercício resistido investigou respostas de pressão arterial ao estresse cardiovascular induzido ${ }^{8}$. Nesse estudo não foram identificadas alterações significativas na reatividade vascular, contudo, o exercício foi realizado com isometria. Sendo assim, existe uma lacuna na literatura sobre possibilidades de aplicação de protocolos de exercícios resistidos isotônicos, que são os mais utilizados na prática clínica e experimental. Além disso, o possível papel do volume destes exercícios, na atenuação da reatividade da pressão arterial ao estresse, também precisa ser investigado. Deste modo, na tentativa de contribuir para o conhecimento acerca do efeito da "dose" de exercícios resistidos na atenuação do estresse cardiovascular, o objetivo do presente estudo foi verificar o efeito do volume da sessão de exercícios resistidos sobre a reatividade da pressão arterial ao estresse induzido.

\section{MATERIAIS E MÉTODOS}

A amostra foi composta por 40 homens, adultos jovens, eutróficos e normotensos (tabela 1). Os critérios de inclusão no estudo foram: 1) Ser praticante de exercícios resistidos há pelo menos seis meses; 2) Não ser tabagista; 3) Não ser usuário de recursos ergogênicos ou de medicamentos que pudessem influenciar as medidas de pressão arterial; 4) Não apresentar disfunções endócrinas, cardiovasculares, ortopédicas ou neurológicas e; 5. Ser do sexo masculino. O tamanho da amostra foi calculado ${ }^{1}$ considerando um Power de 0,80 e o alfa de 0,05 (Gpower 3.1.0 - Franz Faul, Universitat Kiel, Germany). Como resultado, um mínimo de 29 indivíduos para compor o estudo ficou caracterizado. Contudo, prevendo possíveis desistências, um número maior de sujeitos foi selecionado e o estudo foi realizado com 40 participantes, o que possibilitou um maior poder estatístico aos resultados apresentados.

Todos os participantes foram informados previamente sobre os procedimentos de pesquisa e assinaram o Termo de Consentimento Livre e Esclarecido conforme a resolução no 196/96 do Conselho Nacional de Saúde do Brasil. O estudo foi aprovado pelo Comitê de Ética em Pesquisa com Seres Humanos do Hospital São Marcus, em Teresina, Brasil, CAAE - 456075, sob número de protocolo 19/ 2012.

\section{Desenho do Estudo}

Após sessão de teste em dia distinto para mensuração da força máxima (teste de 1RM), os voluntários realizaram randomicamente três sessões experimentais, com intervalo mínimo de 48 horas entre elas e sempre no período da tarde, sendo: 1) Sessão de exercício resistido a 70\% 1RM composta por duas séries (S2); 2) Sessão de exercício resistido a 70\% 1RM composta por quatro séries (S4) e; 3) Sessão controle (SC), sem exercício físico. Ao final de cada sessão experimental um teste de estresse cardiovascular (Cold Pressor Test) fora aplicado para indução da reatividade vascular (RV) de pressão arterial (PA). Mensurações de PA e frequência cardíaca (FC) foram realizadas no repouso pré-sessões experimentais (S2, S4 e SC) e pós-sessões experimentais imediatamente após o Cold Pressor Test.

\section{Procedimentos}

Teste de 1RM: Teste de 1RM para a quantificação das intensidades relativas de exercício de cada participante fora realizado em acordo com os procedimentos descritos por Eng ${ }^{14}$. Os exercícios adotados foram: 1) Supino articulado na máquina vertical; 2) Leg press 450; 3) Desenvolvimento na máquina articulada; 4) Agachamento guiado no hack diagonal; 5) Puxada alta na máquina articulada; 6) Levantamento terra articulado.

Sessões Experimentais: Os participantes foram orientados a não se exercitarem e não ingerirem alimentos ricos em cafeína e bebidas alcoólicas durante um período de no mínimo 48 h prévias as sessões experimentais. Em dias distintos e em ordem randomizada os participantes realizaram duas sessões de exercícios resistidos e uma sessão controle. As sessões de exercício foram uma com duas séries (\$2) e outra com quatro séries (\$4). Ambas foram compostas por seis exercícios (previamente descritos em 1RM), 10 repetições cada, 90 s de intervalo de recuperação entre séries, intensidade de 70\%1RM e velocidade de execução de 1s para a fase concêntrica e 1s para a fase excêntrica. S2 e S4 tiveram duração aproximada de 30 e 50 min, respectivamente. A SC foi realizada no mesmo ambiente das sessões exercício e os indivíduos permaneceram sentados confortavelmente por um período de $40 \mathrm{~min}$.

Tabela 1. Características antropométricas.

\begin{tabular}{c|c}
\hline Variáveis & $\mathbf{N}=\mathbf{4 0}$ \\
\hline Idade $(\mathrm{anos})$ & $25,8 \pm 2,5$ \\
\hline Peso $(\mathrm{Kg})$ & $70,0 \pm 4,2$ \\
\hline Estatura $(\mathrm{m})$ & $1,70 \pm 0,04$ \\
\hline IMC (kg.m²(-1)) & $20,0 \pm 4,3$ \\
\hline \% Gordura & $11,0 \pm 2,0$ \\
\hline Valores são expostos em média e desvio-padrão. IMC: índice de massa corporal.
\end{tabular}


Teste de estresse cardiovascular: Para avaliação da reatividade da pressão arterial (RV) ao estresse, foi utilizado o Cold Pressor Test (CPT), o qual promove um estímulo agudo térmico e, por conseguinte, um reflexo neurogênico com aumento da ativação nervosa simpática ${ }^{15}$, o que pode refletir em aumentos significativos na pressão arterial e frequência cardíaca. ${ }^{12-17}$ O CPT seguiu procedimentos descritos previamente por Kawano et al. ${ }^{18}$ Estes autores demonstraram a reprodutibilidade do CPT em indivíduos normotensos e hipertensos. A especificidade do CPT também foi verificada por Bring e Oerting ${ }^{19}$. O protocolo do CPT solicitou a imersão da mão direita do avaliado (até a altura do punho) em um recipiente com água gelada, com temperatura entre 4-5 C, durante $1 \mathrm{~min}$.

Mensurações de PA e FC foram realizadas nos momentos pré-sessões experimentais (S2, S4 e SC) durante um repouso de 20 min, bem como aos 10 min após as sessões experimentais (S2, S4 e SC) imediatamente após o CPT. Nesse momento, três mensurações de PA sistólica (PAS), diastólica (PAD) e média (PAM) foram realizadas no braço esquerdo do avaliado, utilizando-se um esfigmomanômetro digital (Microlife, modelo BPA 100; China). O valor mais alto foi considerado como o pico de PA. A diferença entre o pico e o repouso da PA foi considerada como a RV ${ }^{15-18}$. Frequência cardíaca (FC) também foi mensurada nos mesmos momentos da PA utilizando-se um cardiofrequencimetro da marca Polar, mod. F1 (Polar Electro Oy, Kempele, Finlândia).

Além disso, imediatamente após as sessões experimentais (S2 e S4) a percepção subjetiva de esforço foi avaliada através da escala de esforço percebido ${ }^{20}$ adaptada de 0 a 10 para o exercício resistido.

\section{Análise estatística}

A variância e a normalidade dos dados foram verificadas pelos testes de Levine e Shapiro-Wilk, respectivamente. Estatística descritiva com procedimentos de média e desvio padrão foi realizada. Teste $t$ Student pareado foi utilizado para comparação dos resultados de FC entre os momentos pré e pós-sessões experimentais. Para analisar o efeito do volume do exercício resistido (SC vs. S2 vs. S4) na FC e PA, foi utilizado a análise de variância ANOVA com post hoc de Tukey. Para todas as análises, o nível de significância adotado foi de $P<0,05$. O software STATISTICA para WINDOWS versão 4.3 (Statsoftlnc., 1993, Tulsa, OK, USA) foi adotado.

\section{RESULTADOS}

Os indivíduos eram normotensos, eutróficos e com idade próxima entre eles. As medidas feitas nos momentos prévios a cada exercício do estudo revelaram que os valores basais de frequência cardíaca e pressão arterial de repouso eram similares entre as três sessões em que os exercícios foram realizados (tabela 2).

Apesar da diferença no volume de séries entre as sessões experimentais de exercício resistido, a percepção subjetiva de esforço ao final foi similar (S2: 8,2 \pm 4,3 vs. S4: 8,2 \pm 4,5 pontos; $p=0,90$ ).

As respostas de FC demonstraram que não ocorreu diferença significativa no repouso pré-exercício entre sessões experimentais ( $p>0,05)$, bem como, imediatamente após o CPT entre sessões experimentais ( $p$ > 0,05). Contudo, após o CPT a FC se mostrou maior quando comparada ao repouso na mesma sessão, especialmente após \$2 e S4 (figura 1; $p<0,05$ ).

A figura 2 apresenta as respostas de RV da PA nas sessões SC, S2 e S4. Verificou-se que o CPT foi eficiente em promover aumentos na

Tabela 2. Características hemodinâmicas prévias à sessão controle (SC), sessão com 2 séries (S2) e sessão com 4 séries (S4).

\begin{tabular}{c|c|c|c}
\hline & SC & S2 & S3 \\
\hline FCR (bpm) & $77 \pm 9$ & $77 \pm 8$ & $79 \pm 6$ \\
\hline PAS $(\mathrm{mmHg})$ & $121 \pm 12$ & $121 \pm 5$ & $121 \pm 4$ \\
\hline PAD $(\mathrm{mmHg})$ & $77 \pm 6$ & $77 \pm 6$ & $77 \pm 6$ \\
\hline PAM (mmHq) & $89 \pm 10$ & $91 \pm 5$ & $91 \pm 4$ \\
\hline
\end{tabular}

Valores em média e desvio-padrão. FCR = Frequência cardíaca de repouso; PAS = Pressão arterial sistólica; PAD = Pressão arterial diastólica; PAM = Pressão Arterial Média.

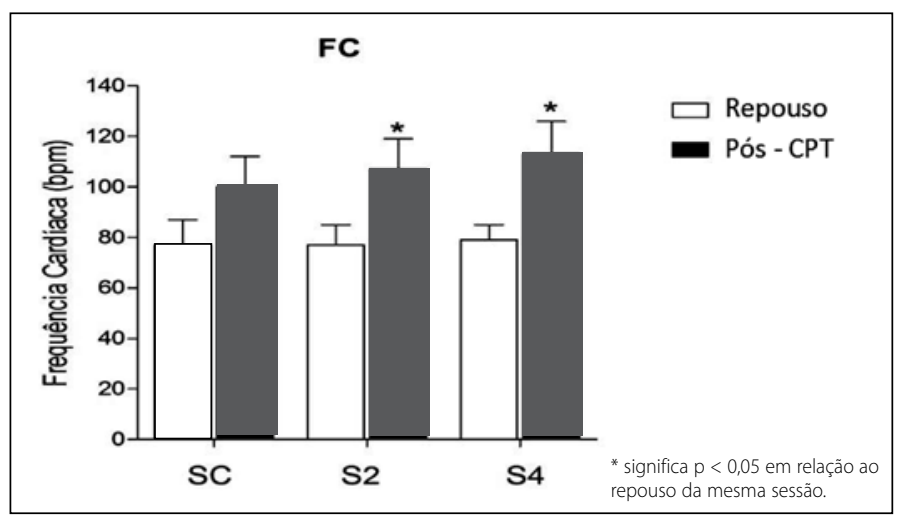

Figura 1. Resposta de frequência cardíaca (FC) no repouso e imediatamente após o Cold Pressor Test (CPT) à sessão controle (SC), sessão com 2 séries (S2) e sessão com 4 séries (S4).

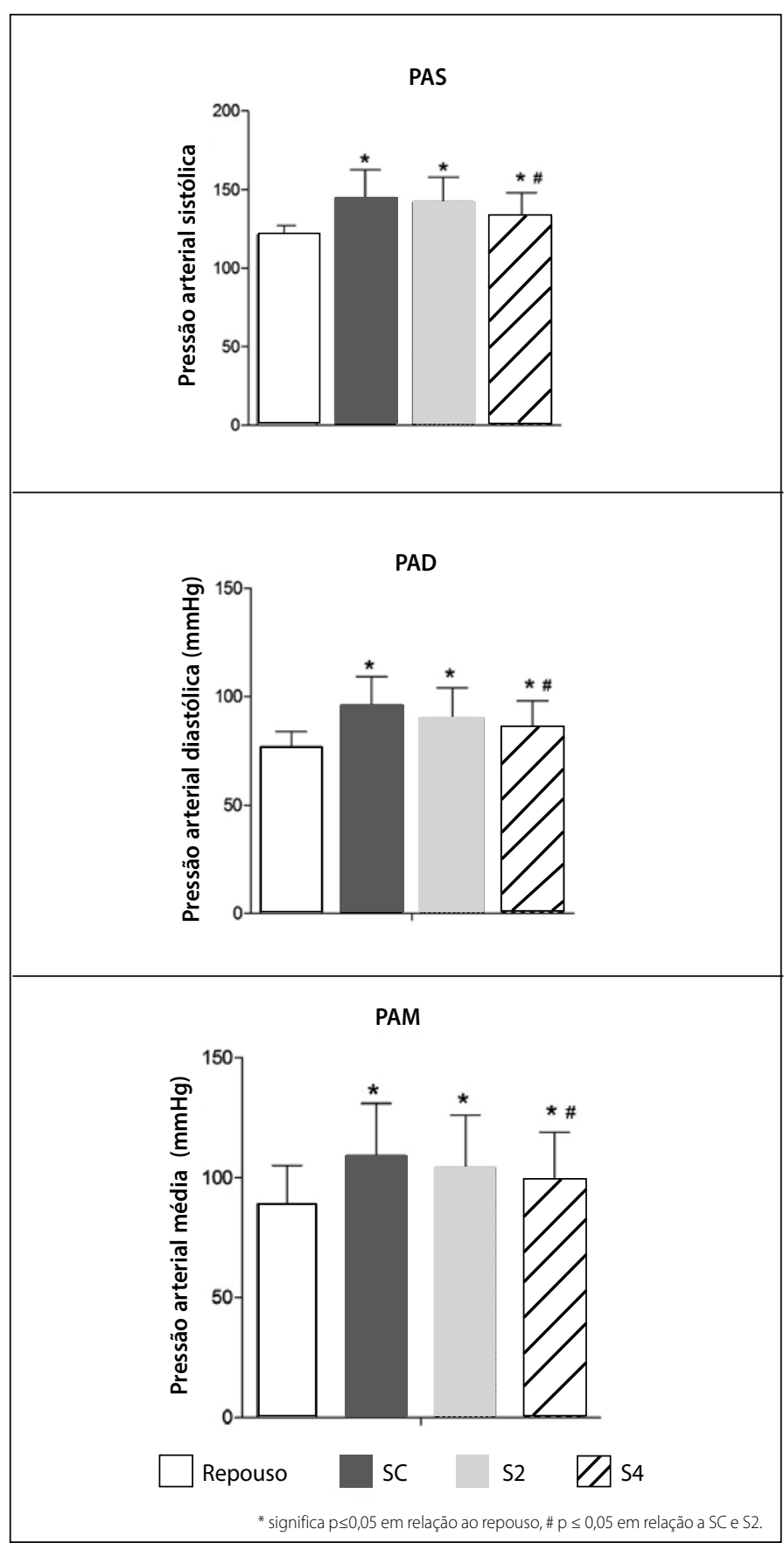

Figura 2. Respostas de pressão arterial sistólica (PAS), diastólica (PAD) e média (PAM) no repouso e imediatamente após o Cold Pressor Test (CPT) à sessão controle (SC), sessão com 2 séries (\$2) e sessão com 4 séries (\$4). 
PAS, PAD e PAM quando comparado ao repouso na mesma sessão ( $p>0,05)$. Entretanto, $S 4$ atenuou a resposta de RV quando comparada em relação às sessões SC e S2 $(p<0,05)$.

\section{DISCUSSÃO}

O principal achado do presente estudo foi que uma única sessão de exercício resistido com quatro, mas não com duas séries de seis exercícios, atenuou a elevação da PA frente a um teste de estresse cardiovascular. Estes resultados indicam que o volume da sessão de exercícios resistidos influencia a reatividade da pressão arterial ao estresse induzido.

Pramanik ${ }^{21}$ destacam que normotensos jovens que apresentaram prolongada elevação na PAD em resposta à estimulação simpática induzida pelo CPT, estão propensos a desenvolver hipertensão. Nesse sentido, mesmo que os sujeitos da presente investigação não sejam hipertensos, avaliar a resposta pressórica frente a um desafio cardiovascular, bem como, a influência do volume do exercício resistido nessa resposta, pode ter importante aplicação clínica e prática na área da saúde voltada a prescrição do exercício físico.

Algumas alternativas já foram apresentadas na literatura como ferramentas de atenuação da resposta pressórica ao CPT, sendo: 1) Suplementação oral com L-citrulina22; 2) Aplicação de acupuntura 23; 3) Uso de fármacos ${ }^{24}$ e; 4) Prática de exercícios aeróbios ${ }^{25}$. Quanto ao exercício resistido isotônico à 70\%1RM, em nosso conhecimento este é o primeiro estudo a apresentar resultados consistentes de atenuação da resposta pressórica induzida pelo CPT e sendo esta influenciada pelo volume da sessão de exercício resistido. Outros estudos, investigando o efeito da intensidade do exercício resistido, bem como, de sessões combinadas de exercício aeróbio+resistido nas respostas de RV ainda são necessários.

Richter et al. ${ }^{3}$ ao investigarem normotensos com hiperreatividade em um estudo com treinamento aeróbio, demonstrou reduções em torno de $8 \pm 16 \mathrm{mmHg}$ na PAS e $3 \pm 5 \mathrm{mmHg}$ na PAD. No presente estudo, os valores de atenuação da PAS para as sessões S2 e S4 foram de $2 \pm 16 \mathrm{mmHg}$ e $9 \pm 15 \mathrm{mmHg}$, respectivamente. Na PAD ocorreram reduções de $6 \pm 14 \mathrm{mmHg}$ e $10 \pm 12 \mathrm{mmHg}$, para S2 e S4, respectivamente. Estes resultados sugerem que sessões agudas de exercício resistido podem ser tão eficazes, na proteção cardiovascular, quanto às respostas

\section{REFERÊNCIAS}

1. Vanitallie TB. Stress: a risk factor for serious illness. Metabolism. 2002;51(6 Suppl 1):40-5.

2. Vatner $S F$, Vatner $D E$, Homcy CJ. beta-adrenergic receptor signaling: an acute compensatory adjustment-inappropriate for the chronic stress of heart failure? Insights from Gsalpha overexpression and other genetically engineered animal models. Circ Res. 2000;86(5):502-6.

3. Richter CM, Panigas TF, Bündchen DC, Dipp T, Belli KC, Viecili PR. Blood pressure reduction in hyper-reactive individuals after aerobic exercise. Arq Bras Cardiol. 2010;95(2):251-7.

4. Probst M, Bulbulian R, Knapp C. Hemodynamic responses to the stroop and cold pressor tests after submaximal cycling exercise in normotensive males. Physiol Behav. 1997;62(6):1283-90.

5. Gerin W, Zawadzki MJ, Brosschot JF, Thayer JF, Christenfeld NJ, Campbell TS, et al. Rumination as a mediator of chronic stress effects on hypertension: a causal model. Int J Hypertens. 2012;2012:453465.

6. Hines EA Jr, Brown GE. The cold pressor test for measuring the reactibility of the blood pressure: data concerning 571 normal and hypertensive subjects. Am Heart J. 1936;11:1-9.

7. Wood DL, Sheps SG, Elveback LR, Schirger A. Cold pressor test as a predictor of hypertension. Hypertension. 1984;6(3):301-6.

8. Kimmerly DS, O'Leary DD, Menon RS, Gati JS, Shoemaker JK. Cortical regions associated with autonomic cardiovascular regulation during lower body negative pressure in humans. J Physiol. 2005;569(Pt 1):331-45

9. Kobori H, Fu Q, Crowley SD, Gonzalez-Villalobos RA, Campos RR. Comments on Point:Counterpoint: The dominant contributor to systemic hypertension: Chronic activation of the sympathetic nervous system vs. Activation of the intrarenal renin-angiotensin system. Activated intrarenal renin-angiotensin system is correlated with high blood pressure in humans. J Appl Physiol (1985). 2010;109(6):2003-14

10. Gaesser GA, Angadi SS, Sawyer BJ. Exercise and diet, independent of weight loss, improve cardiometabolic risk profile in overweight and obese individuals. Phys Sportsmed. 2011;39(2):87-97.

11. Brito AF, Alves NF, Araújo AS, Gonçalves MC, Silva AS. Active intervals between sets of resistance exercises potentiate the magnitude of postexercise hypotension in elderly hypertensive women. J Strength Cond Res. 2011;25(11):3129-36.

12. Costa RA, Nascimento RL, Souza JA, Almeida CM, Silva AA, Carvalho FO, et al. Post-Exercise Hypotension Attenuates The Effect of Waist on Vascular Reactivity in Adults of Both Sexes. JEPOnline. 2012;15(1):87-97.

13. Ullman B, Lindvall K, Lundberg JM, Sigurdsson A, Swedberg K. Response of plasma neuropeptide $\mathrm{Y}$ and noradrenaline to dynamic exercise and ramipril treatment in patients with congestive heart failure. Clin Physiol. 1994;14(2):123-34. crônicas ao treinamento aeróbio. Contudo, a magnitude dos efeitos aqui apresentados pode perdurar apenas ao longo das horas subsequentes à realização da sessão, o que nos leva a sugerir que o exercício resistido também seja realizado cronicamente, especialmente para promover outros benefícios à saúde (morfológicos, metabólicos e hemodinâmicos), os quais essa modalidade pode oferecer.

Apesar da presente inquérito não ter como objetivo investigar os mecanismos candidatos na atenuação da RV induzida, outras evidências sugerem a ocorrência, nos momentos agudos da recuperação pós-exercício, de uma maior biodisponibilidade de óxido nítrico ${ }^{18}$, reduzida sensibilidade da ativação nervosa simpática ${ }^{26}$ e aumento nas endorfinas circulantes ${ }^{27}$. Esses resultados poderiam explicar as respostas de atenuação da RV de PA após uma única série de exercício resistido, como constatado no presente estudo.

Uma das limitações do presente estudo a ser discutida poderia ser a não mensuração do estresse cardiovascular durante as atividades da vida diária da amostra, o que poderia trazer implicações clínicas importantes. Contudo, o teste de estresse cardiovascular utilizado (CPT) foi eficiente para induzir a RV de PA nos momentos da recuperação pós-sessões experimentais (figura 2).

\section{CONCLUSÃO}

Concluímos que o exercício resistido pode atenuar a reatividade da pressão arterial ao estresse subsequente, e que o volume realizado desempenha um papel nesta atenuação. No presente estudo apenas a sessão de maior volume de ER (4 séries vs. 2 séries) resultou em atenuação da reatividade da PA frente ao estresse induzido. Novas investigações são necessárias através da monitoração ambulatorial da PA durante períodos prolongados (ex. 24 h), com objetivo de investigar a magnitude da resposta protetora do exercício físico frente ao estresse cardiovascular da vida diária. Finalmente, são necessários estudos sobre os possíveis mecanismos envolvidos nesta atenuação da reatividade da pressão arterial ao estresse, promovida pelo exercício resistido prévio.

Todos os autores declararam não haver qualquer potencial conflito de interesses referente a este artigo.
14. Eng J. Sample size estimation: how many individuals should be studied? Radiology. 2003;227(2):309-13.

15. Harbach H, Hell K, Gramsch C, Katz N, Hempelmann G, Teschemacher H. Beta-endorphin (1-31) in the plasma of male volunteers undergoing physical exercise. Psychoneuroendocrinology. 2000;25(6):551-62.

16. Fahs CA, Heffernan KS, Fernhall B. Hemodynamic and vascular response to resistance exercise with L-arginine. Med Sci Sports Exerc. 2009, 41(4):773-9.

17. Wang L, Hou L, Li H, Chen J, Kelly TN, Jaquish CE, et al. Genetic variants in the renin-angiotensin system and blood pressure reactions to the cold pressor test. J Hypertens. 2010;28(12):2422-8.

18. Kawano H, Tanimoto M, Yamamoto K, Sanada K, Gando Y, Tabata I, et al. Resistance training in men is associated with increased arterial stiffness and blood pressure but does not adversely affect endothelial function as measured by arterial reactivity to the cold pressor test. Exp Physiol. 2008;93(2):296-302.

19. Bring JF, Oerting, H. Vasomotor response of normal and hypertensive individuals to standart stimulus (cold). Minnesota Med. 1933;16:481.

20. Borg GA. Psychophysical bases of perceived exertion. Med Sci Sports Exerc. 1982;14(5):377-81.

21. Pramanik T, Regmi P, Adhikari P, Roychowdhury P. Cold Pressor Test as a Predictor of Hypertension. J Teh Univ Heart Ctr. 2009;3:177-80.

22. Figueroa A, Trivino JA, Sanchez-Gonzalez MA, Vicil F. Oral L-citrulline supplementation attenuates blood pressure response to cold pressor test in young men. Am J Hypertens. 2010;23(1):12-6.

23. Pautasso EJ, Koretzky M, Aiub J, Fovemtsac R, Borregomtsac C, Cecco FD, et al. Is Cold Pressor Test Useful to Predict Cardiovascular Events in Patients with Not Documented Coronary Artery Disease? Rev Arg Cardiol. 2010;78(5):417-23.

24. Earle RW. Weight training exercice prescrition. In: Baechle TR, Earle RW. Essential of personal training symposium workbook. Lincon, NE: NSCA Certification Commission; 2006. p.1-41.

25. Bond V, Mills RM, Caprarola M, Vaccaro P, Adams RG, Blakely R, et al. Aerobic exercise attenuates blood pressure reactivity to cold pressor test in normotensive, young adult African-American women. Ethn Dis. 1999;9(1):104-10.

26. Rezk CC, Marrache RC, Tinucci T, Mion D Jr, Forjaz CL. Post-resistance exercise hypotension, hemodynamics, and heart rate variability: influence of exercise intensity. Eur J Appl Physiol. 2006;98(1):105-12.

27. Kraemer WJ, Dziados JE, Marchitelli LJ, Gordon SE, Harman EA, Mello R, et al. Effects of different heavy-resistance exercise protocols on plasma beta-endorphin concentrations. J Appl Physiol (1985). 1993;74(1):450-9 